\title{
Did you say a BLUE banana? The prosody of contrast and abnormality in Bulgarian and Dutch
}

\author{
Diana V. Dimitrova ${ }^{1}$, Gisela Redeker ${ }^{1}$, John C.J. Hoeks ${ }^{1}$ \\ ${ }^{1}$ Center for Language and Cognition, University of Groningen, The Netherlands \\ d.dimitrova@rug.nl, g.redeker@rug.nl, j.c.j.hoeks@rug.nl
}

\begin{abstract}
In a production experiment on Bulgarian that was based on a previous study on Dutch [1], we investigated the role of prosody when linguistic and extra-linguistic information coincide or contradict. Speakers described abnormally colored fruits in conditions where contrastive focus and discourse relations were varied. We found that the coincidence of contrast and abnormality enhances accentuation in Bulgarian as it did in Dutch. Surprisingly, when both factors are in conflict, the prosodic prominence of abnormality often overruled focus accentuation in both Bulgarian and Dutch, though the languages also show marked differences.

Index Terms: prosody, semantic abnormality, contrast,
\end{abstract} Bulgarian, Dutch

\section{Introduction}

Prosody refers to both speech melody and rhythm and is claimed to signal extra-linguistic information such as speaker's attitude as well as linguistic functions such as information structure. The extra-linguistic functions of prosody are claimed to be language independent and can possibly be attributed to a set of biological codes [2]. In contrast, linguistic functions of prosody appear to be specific for Germanic languages such as Dutch and English [3]. However, little is known about the functions of intonation in Slavic languages such as Bulgarian [4]

One of the well-studied linguistic functions of prosody is the indication of focus, i.e., the most informative part of a message. Different types of pitch accents have been attributed to the marking of various types of focus. Moreover, it has been suggested that contrastive focus exhibits a specific pitch accent (i.e., $\mathrm{L}+\mathrm{H}^{*}$ ) [5]. Studies on Dutch, however, failed to identify a specific type of contrastive pitch accent [6]. Nevertheless, it is assumed that contrastive prosody exists: a contrastive interpretation arises on the basis of a non-default position of the most prominent (i.e., nuclear) pitch accent.

In addition, English speakers use prosody to signal semantically abnormal information [7]. Since in this study, abnormal information is defined as a violation of contextual expectations, prosody can be viewed as guiding the attention of the listener to relevant information [8].

Research on the interaction between linguistic and extralinguistic functions of prosody is scarce. This study aims to provide experimental evidence for the speaker's preferences for accentuation when linguistic contrast and extra-linguistic abnormality coincide or are in conflict.

\section{Contrast and Prosody in Dutch}

In Dutch, prosodic prominence is assigned in accordance with information structure: focused elements are accented, and background information is deaccented. Moreover, the strength of prosodic prominence has been found to vary depending on which element of the noun phrase (NP) is contrasted [9]. In general, contrasted adjectives are more likely to bear single matching accents than nouns are. Even though contrasted nouns are assigned single accents as well, they are also likely to get double accents (i.e., both NP elements are accented). Since the hypotheses of our current experiment are based on the outcome of this previous research on the accentuation of contrast in Dutch [1,9], we will discuss it in further detail.

In the original experiment [9], participants were asked to describe scenes consisting of geometrical figures which varied in color and which were involved in various contrastive relations. The results suggest that discourse impacts the prosody of contrasted adjectives and nouns. Accentuation of contrast was significantly stronger when the contrasted element occurred in final sentence position (i.e., backward contrast relation) as compared to initial sentence position (i.e., forward contrast relation) (cf. (1)). This factor, contrast direction, applied to contrasts within the sentence boundaries and affected the prosody of both adjectives and nouns. Moreover, differences in accentuation also arose due to the syntactic status of NP elements: contrast in object position triggered more corresponding single accents than did contrast in subject position (cf. (2)). Finally, discourse distance influenced the accentuation of contrastive focus in such a way that contrasts within the sentence (e.g., (1)) were prosodically stronger than contrasts across the sentence (e.g., (2)). Note that in all examples, both elements in a contrastive set are italicized, and target elements are presented in capitals.

(1) The red square touches the BLUE square.

(2) The green triangle touches the red square. The green triangle touches the BLUE square.

In our version of this study, we introduced semantic abnormality as an additional extra-linguistic factor.

\section{Abnormality and Prosody in Dutch}

In our previous study on Dutch [1], we modified the semantic content of the stimuli in [9] in order to introduce abnormality as an additional extra-linguistic factor. We replaced the geometrical figures in [9] by fruits that always occurred in an unnatural color. Thus, abnormal information (e.g., a blue banana) mismatched a conceptual representation (e.g., a yellow banana). As a result, this modification to the original design introduced: (1) a prominence conflict where both factors worked in opposite directions (i.e., abnormality triggered prominence of the adjectives whereas contrastive focus increased the salience of nouns); or (2) a prominence coincidence where both factors worked in the same direction (i.e., abnormality and contrastive focus applied both to adjectives). We found that semantic abnormality often reverses the prosodic patterns for marking of contrastive 
focus in Dutch. Regardless of which element has been contrasted, adjectives were most often accented. Furthermore, semantic abnormality appeared superior to contextual effects. Except for discourse distance, the discourse factors tested did not significantly affect the prosodic realization of contrastive focus. Our findings thus provide evidence for the importance of extra-linguistic information for accentuation.

In the current experiment, we investigate whether we will obtain the same effects in Bulgarian.

\section{Prosody in Bulgarian}

Research on the linguistic functions of prosody in Bulgarian has mostly concentrated on the phonetic characteristics of sentence types [10]. A few recent studies have addressed the prosodic correlates of information structural categories $[4,11]$. Their results suggest that focus is prosodically marked in Bulgarian by means of pitch accents. However, there is no phonological difference between the accentuation of contrastive and non-contrastive focus [11].

To our knowledge, no studies so far have investigated whether focused adjectives behave prosodically differently as compared to focused nouns in Bulgarian. Moreover, it is unclear if and how the accentuation of focus is affected by discourse in this language. There is no evidence if speakers of Bulgarian perceive contrastive accentuation as distinct from a non-contrastive one. However, it has been suggested that strong prosodic prominence is necessary for contrastive focus, especially in initial sentence position [12]. Even further, no studies have investigated how abnormal information is prosodically marked in Bulgarian.

\section{The Experiment}

\subsection{Participants}

Ten native speakers of Bulgarian (age 19-33; four female) volunteered to participate in a production study that lasted approximately 15 minutes.

\subsection{Procedure}

All recordings were made in a home setting in Bulgaria. Participants were presented with various scenes on a computer screen that consisted of three successive movements. Each scene displayed four pairs of fruits (bananas, lemons, cherries, and raspberries) in an unnatural color (e.g., blue, grey, etc.). The participants initiated the first movement with a mouse click and as a result, one pair of fruits moved towards another one, touched it, and returned to its original position. After the movement was completed, participants provided a description of the action. They were asked to name both the color and the type of fruit by producing sentences with a fixed SVO word order such as "The red bananas touch the blue bananas on the screen". We included a prepositional phrase after the object NP to rule out a possible combination of nuclear pitch accents with boundary tones in sentence final positions as well as to limit syntactic variation. The actual experiment was preceded by a practice session.

\subsection{Materials}

Figure 1 displays two experimental scenes in which consecutive movements are indicated by numbered arrows. Colors are displayed on a grayscale and target sentences are presented in a box.

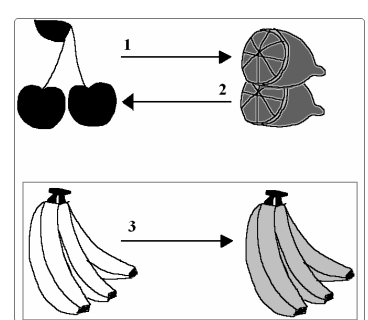

(a)

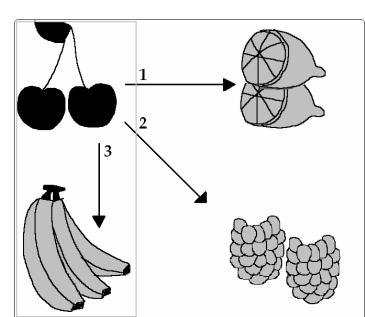

(b)
Figure 1: Experimental scenes: (a) contrasted adjectives within the sentence (red vs. grey bananas); (b) contrasted nouns across the sentence (blue bananas vs. blue lemons).

The first two actions of a scene (cf., Figure 1, arrows 1 and 2) set up a discourse context for the target sentence which represents the third (and last) movement in a scene. Two NPs were defined as targets ("sinite limoni", the blue lemons and "sivite banani", the grey bananas).

\section{Contrast Direction}

early contrast The GREY bananas touch the red bananas.
late contrast The red bananas touch the GREY bananas.

\section{Syntactic Status}

subject contrast

The red bananas touch the blue cherries.

The GREY bananas touch the blue cherries.

object contrast The blue cherries touch the red bananas. The blue cherries touch the GREY bananas.

\section{Discourse Distance}

within contrast The red bananas touch the GREY bananas. across contrast The blue cherries touch the red bananas. The blue cherries touch the GREY bananas.

Table 1. Experimental conditions.

Various types of contrast relations were established in each target sentence. First, the contrasted item was either the color of identical types of fruits (cf. Figure 1a) or the type of fruits with identical colors (cf. Figure 1b). Second, the contrasted target NPs occurred either in an early or in a late contrast (e.g., the first or the last mentioned element of a contrastive pair, respectively). Third, a contrasted element was coded as either a subject or as an object. Fourth, the NP elements were contrasted either within the target sentence or across the sentence boundary (e.g., between the target NP and an NP in the preceding second sentence). These discourse factors have been found to affect the accentuation of contrastive focus [9]. All conditions are listed in Table 1.

\subsection{Hypothesis}

In line with $[4,11,12]$ we predict that contrastive focus in Bulgarian should be realized by means of accentuation. Due to the lack of research on the prosodic patterns of elements within the NP as well as on abnormality and discourse, our study has an exploratory character.

\subsection{Analysis}

From all 240 recorded sentences (24 target sentences x 10 subjects), $4(0.6 \%)$ were excluded from further analysis due to errors and hesitations. Two intonation experts (the first author and an independent intonation researcher) performed an auditory analysis of target NP elements. The labelers judged the prosodic prominence: they annotated items that stood out 
perceptually as most prominent due to a higher pitch, a pitch accent, a higher intensity, or a combination thereof. Perceptually prominent elements are referred to as accented elements. We did not perform a phonological analysis of pitch accent types which we will address in future research.

\subsection{Results}

Mean percentages Accented Element (adjective vs. noun vs. both) were calculated in each of the three major sets of conditions: 1) contrast direction, 2) syntactic status of contrasted element, 3) discourse distance between contrasted elements. See Table 2 for actual percentages in all (sub-) conditions. We conducted Repeated Measures ANOVAs for the three major condition sets separately, each with three within-subjects factors: Accented Element (adjective vs. noun vs. both); Contrasted Element (adjective vs. noun), together with one of the following factors that are unique to a given condition set: Contrast Direction (early vs. late), Syntactic Status (subject vs. object), Discourse Distance (within sentence vs. across sentence). In order to compare these results with the previously collected Dutch results [1], we carried out the analyses mentioned above with the additional betweensubjects factor Language (Bulgarian vs. Dutch). All statistical tests are performed on transformed values (using arcsine transformation), and all post-hoc comparisons are Bonferronicorrected.

\subsubsection{Contrast Direction}

The factor Contrast Direction did not have a significant effect on the accentuation of contrast. There was a main effect of Accented Element $(\mathrm{F}(2,18)=7.7, \mathrm{p}<.05)$ which was qualified by a significant interaction between Accented Element and Contrasted Element $(\mathrm{F}(2,18)=12.6, \mathrm{p}<.001)$. Post-hoc tests showed that this interaction results from a significant effect of Accented Element for adjectives $(\mathrm{p}<.001)$, but not for nouns $(\mathrm{p}=.12)$. If the contrasted element was an adjective, there were significantly more accented adjectives $(77.5 \%$; $\mathrm{SE}=8.7)$ than nouns $(15 \%$; $\mathrm{SE}=5.5)$ or accents on both $(7.5 \%$; $\mathrm{SE}=5.3)$. If, however, the noun was contrasted, there was no corresponding increase in accented nouns $(40 \%$; $\mathrm{SE}=11.3)$; numerically (but not significantly), there were even more accented adjectives $(50 \% ; \mathrm{SE}=12.9)$.

When comparing Bulgarian to Dutch, we found no significant interactions involving language. Thus, in both languages, abnormality overruled the accentuation of contrast.

\subsubsection{Syntactic Status}

The factor Syntactic Status was involved in a significant threeway interaction with Contrasted Element and Accented Element $(\mathrm{F}(2,18)=5.0, \mathrm{p}<.05)$. Follow-up analyses showed an interaction of Contrasted Element with Accented Element for grammatical subjects $(\mathrm{p}<.01)$, but no such interaction for grammatical objects $(\mathrm{p}=.11)$. Further investigating the data for grammatical subjects, we document a main effect of Accented Element for adjectives $(\mathrm{p}<.05)$, but not for nouns $(\mathrm{p}=.24)$, showing the same pattern as described above. When adjectives are contrasted, a significant increase in accents on the adjectives is observed, as compared to the increase in nouns. When nouns are contrasted, we did not find an increase in accentuation, and even observed a numerical advantage for accented adjectives.

As far as the language tested is concerned, we observed a four-way interaction Syntactic Status x Contrasted Element $\mathrm{x}$ Accented Element $\mathrm{x}$ Language. For grammatical subjects, there was no effect involving Language $(\mathrm{F}<1)$, indicating that Bulgarian and Dutch show highly similar prosodic patterns for contrast in subject position. Regardless of contrasted element, adjectives are most prominent. On the contrary, responses differed between languages when grammatical objects were concerned. Such language disparity was only apparent for nouns and there was no effect involving language for adjectives. Hence, in object position, the effect of anomaly is very strong in Bulgarian: there are significantly more accents on adjectives even though nouns are contrasted. The opposite is true for Dutch: if nouns are contrasted in object position, they are prosodically more prominent than adjectives. No other differences between the two languages were significant.

\begin{tabular}{|c|c|c|c|c|c|c|c|}
\hline \multirow{3}{*}{$\begin{array}{l}\text { Contrast } \\
\text { Type }\end{array}$} & \multirow{3}{*}{ Element } & \multicolumn{4}{|c|}{ ACCENTED ELEMENT } & \multirow{2}{*}{\multicolumn{2}{|c|}{ Both }} \\
\hline & & \multicolumn{2}{|c|}{ Adjective } & \multicolumn{2}{|c|}{ Noun } & & \\
\hline & & $\%$ & $S E$ & $\%$ & $S E$ & $\%$ & $S E$ \\
\hline \multicolumn{8}{|c|}{ Contrast Direction } \\
\hline \multirow[t]{2}{*}{ Early } & Adjective & 70 & 13.3 & 25 & 11.2 & 5 & 5.0 \\
\hline & Noun & 55 & 13.8 & 40 & 12.5 & 5 & 5.0 \\
\hline \multirow[t]{2}{*}{ Late } & Adjective & 85 & 10.7 & 5 & 5.0 & 10 & 10.0 \\
\hline & Noun & 45 & 13.8 & 40 & 12.5 & 15 & 7.6 \\
\hline \multicolumn{8}{|c|}{ Syntactic Status } \\
\hline \multirow[t]{2}{*}{ Subject } & Adjective & 85 & 10.7 & 10 & 10.0 & 5 & 5.0 \\
\hline & Noun & 35 & 13.0 & 50 & 12.9 & 15 & 7.6 \\
\hline \multirow[t]{2}{*}{ Object } & Adjective & 80 & 11.1 & 5 & 5.0 & 15 & 10.7 \\
\hline & Noun & 60 & 14.5 & 10 & 6.7 & 30 & 11.0 \\
\hline \multicolumn{8}{|c|}{ Discourse Distance } \\
\hline \multirow[t]{2}{*}{ Within } & Adjective & 85 & 10.7 & 5 & 5.0 & 10 & 10.0 \\
\hline & Noun & 45 & 13.8 & 40 & 12.5 & 15 & 7.6 \\
\hline \multirow[t]{2}{*}{ Across } & Adjective & 80 & 11.0 & 5 & 5.0 & 15 & 10.7 \\
\hline & Noun & 60 & 14.5 & 10 & 6.7 & 30 & 11.0 \\
\hline
\end{tabular}

Table 2. Accentuation percentages (plus SE) for all (sub-) conditions in each of the major conditions.

\subsubsection{Discourse Distance}

We found a significant three-way interaction of Discourse Distance $x$ Contrasted element $x$ Accented Element. Follow-up analyses exhibit a significant interaction of Contrasted Element $x$ Accented Element for contrast within the sentence $(\mathrm{p}<.001)$ but no such interaction for contrast across the sentence $(\mathrm{p}=.11)$. For within-contrast, there was a marginally significant effect of Accented Element for contrasted nouns $(\mathrm{p}=10)$, and a highly significant effect for contrasted adjectives, indicating that in both cases there were more accents on adjectives than on nouns.

When comparing Dutch and Bulgarian, we note a fourway interaction Discourse Distance x Contrasted Element $\mathrm{x}$ Accented Element x Language. The interaction Contrasted Element $\mathrm{x}$ Accented Element with Language was found only for contrasts across the sentence boundary but not for contrasts within the sentence. Follow-up analyses for across cases showed essentially the same pattern that we saw for Syntactic Status, namely a strong anomaly effect in Bulgarian where there were more accents on adjectives when nouns were contrasted. Dutch showed a reverse 'normal' pattern, with significantly more accents on nouns when nouns were contrasted across the sentence boundary.

\section{Discussion}

We found that linguistic as well as extra-linguistic factors are prosodically marked in both Bulgarian and Dutch. When these 
factors are in conflict, prosody often reflects semantic abnormality rather than contrastive focus in both languages.

\subsection{Discourse and Prosody}

Similar to our results for Dutch [1] and opposite to [9], contrast direction did not affect accentuation in Bulgarian. We showed that contrasted elements are accented equally often in early and late positions and regardless of whether they are contrasted or not. We propose that the overall prosodic prominence of adjectives might be caused by semantic abnormality. The same patterns apply for discourse distance in Bulgarian but not in Dutch. In Bulgarian, irrespective of focus domain and the distance between contrasted elements, adjectives are prosodically most prominent. In Dutch, accents are assigned in line with focus only if elements are contrasted across the sentence. In a previous study [1], we suggested that repetition of abnormality might diminish its impact on prosody. However, this interpretation should be revised because repetition did not affect accentuation in Bulgarian even though deaccentuation of repeated information is common for Bulgarian [4]. It is unclear why the linguistic functions of prosody overrule the marking of abnormality in object position in Dutch, and in subject position in Bulgarian. Only in subject position in Bulgarian is prosody assigned in line with contrastive focus when the syntactic status of the elements is concerned. The fact that Bulgarian is a pro-drop language provides further support for the assumption that the realization of a grammatical subject is highly prominent. Further research on the topic should shed more light on the interactions between syntax and prosody in both languages.

\subsection{Semantic Abnormality and Prosody}

Semantic abnormality was defined in terms of a violation of conceptual representations. The identification of abnormality requires a comparison of abnormal information with memory representations and a mismatch detection.

Yet, one might argue that using unnatural colors in line drawings of fruit does not constitute a real semantic abnormality: The unnatural colors of fruits may not undergo a conceptual check but be rather treated by the speakers as pure illustrations. Moreover, speakers may get used to the abnormal objects as the experiment proceeds and even form expectations for unnatural fruits. In such a scenario, nothing would count as 'abnormal' since memory representations of real world objects are not activated. Thus, our abnormal fruits should be as normal as geometrical figures that lack any color association. In that case, however, we should have replicated the prosodic patterns for Dutch in [9], which we did not [1]. At this point, we believe that speakers perceive the unnatural colors of the line drawing as anomalous and use prosody to signal this abnormality, even at the expense of the communicative function of expressing contrastive focus [1].

In this paper, we provided evidence that semantic abnormality should be taken into account when considering the extra-linguistic functions of prosody. The impact of conceptual violations on language processing has been attested in various perception and neuroimaging studies. It is claimed that perception is driven by memory [13]: participants' memory for a real world object (e.g., a yellow banana) influences perception (e.g., a grey banana still appears yellowish). In addition, neuroimaging studies report distinct underlying neural processing structures for semantic violations as opposed to semantic matches (e.g., sour trains vs. yellow trains) [14]. Furthermore, neuroimaging research suggests that semantic mismatches are processed earlier in time than are syntactic violations. Thus, the neural processing of extralinguistic information precedes the processing of linguistic information. On the production level, thus far no experiments have systematically attested how semantic mismatches are realized in spoken language. Our study suggests that the signaling of extra-linguistic over linguistic information may hold for language production as well, with prosody being only one possible tool for its realization. Future research should provide deeper insights in the role of prosody for indicating conceptual violations.

\section{Conclusions}

Throughout this study, we showed that linguistic and extralinguistic information are both expressed prosodically. In Bulgarian as well as in Dutch, semantic abnormality overrules the accentuation of contrastive focus and reduces the impact of discourse. This is as yet another illustration of the profound influence of world knowledge on language processing.

\section{Acknowledgements}

We are very grateful to Maartje Schreuder for her help with the analysis of the stimulus materials.

\section{References}

[1] Dimitrova, D., Redeker, G., Egg, K.M.M. and Hoeks, J.C.J., "Prosodic correlates of linguistic and extra-linguistic information in Dutch", in B. C. Love, K. McRae and V. M. Sloutsky [Eds.], Proceedings of $\mathrm{CogSCi} 2008$, Austin, TX: Cognitive Science Society, 2191-2196, 2008.

[2] Gussenhoven, C., The Phonology of tone and intonation. Cambridge: Cambridge University Press, 2004

[3] Vallduv1, E., "The role of plasticity in the association of focus and prominence". In Proceedings of the ESCOL, 295-306, 1991.

[4] Andreeva, B., Avgustinova, T. and Barry, W.J., "Linkassociated and focus-associated accent patterns in Bulgarian", in G. Zybatow, U. Junghanns, G. Mehlhorn and L. Szucsich [Eds.], Current Issues in Formal Slavic Linguistics, Frankfurt: Lang, 353-364, 2001

[5] Pierrehumbert, J. and Hirschberg, J., "The meaning of intonation in the interpretation of discourse", in P. Cohen, J. Morgan and M. Pollack [Eds.], Intentions of communication, Cambridge: MIT, 271-311, 1990

[6] Krahmer, E. J. and Swerts, M., "On the alleged existence of contrastive accents". Speech Communication, 34: 391-405, 2001.

[7] Pan, S., McKeown, K. and Hirschberg, J., "Semantic abnormality and its realization in spoken language", EUROSPEECH, 333-336, 2001.

[8] Wilson, D. and Wharton, T., "Relevance and prosody", Journal of Pragmatics, 38: 1559-1579, 2006.

[9] Swerts, M., "Contrast and accent in Dutch and Romanian", Journal of Phonetics, 35: 380-397, 2007.

[10] Miševa, A., Intonacionna sistema na bălgarskija ezik. Sofija: Bălgarska Akademija na Naukite, 1991.

[11] Andreeva, B., Zur Phonetik und Phonologie der Intonation in der Sofia Varietät des Bulgarischen, PHONUS 12, Saarbrücken, $\mathrm{PhD}$ thesis, 2007.

[12] Stanchev, S.B., "Pragmatic functions and special sentence positions in Bulgarian", in J.H. Connolly, R.M. Vismans, C.S. Butler and R.M. Gatward [Eds.], Discourse and pragmatics in functional grammar, Berlin: Gruyter, 121-137, 1997.

[13] Hansen, T., Olkkonen, M., Walter, S. and Gegenfurtner, K. R., "Memory modulates color appearance", Nature Neuroscience, 9(11): 1367-1368, 2006

[14] Hagoort, P., Hald, L., Bastiaansen, M. and Petersson, K. M., "Integration of word meaning and world knowledge in language comprehension", Science, 304: 438-441, 2004. 